\title{
Mobile Behavioral Therapy for Headache: Pilot Study
}

\author{
Adam Kirell; Rebecca Shingleton, Dr PhD \\ BioTrak Therapeutics, New York, NY, United States
}

\section{Corresponding Author:}

Adam Kirell

BioTrak Therapeutics

320 E 65th Street

New York, NY,

United States

Phone: 5241934

Email:

\begin{abstract}
Background: Frequently recurring tension headaches, migraines, and orofacial pain affect over 2.4 billion people worldwide, representing the second most prevalent medical condition in the world. In-office behavioral therapy (ie, relaxation and mindfulness exercises) has been shown to be one of the most effective treatment options for these patients. However, it has been underutilized due to stigma, cost, and accessibility. Mobile health apps may be one way to provide accessible, lower cost care in a nonstigmatized environment. As a result, apps are becoming an increasingly popular method for delivering behavioral therapy interventions and may provide an effective means to reach this population.

Objective: The aim of this study was to assess the feasibility and acceptability of delivering a clinically validated behavioral therapy for migraine/headaches through a mobile app.

Methods: Twenty-one migraine or headache sufferers met the criteria for severe migraine disability determined by the MIDAS test. Participants completed a two-week baseline period in which they kept a daily headache diary through the Halo app. They then began a four-week intervention period. During this period they were instructed to complete ten minutes of relaxation training exercises provided by the app each day, as well as monitor headaches via the daily headache diary. Relaxation training exercises consisted of various breathing techniques, progressive muscle relaxation, and mindfulness. Headache frequency was tracked throughout the intervention period, and further assessments, such as an in-person interview and posttreatment MIDAS test, were completed at the end of the four-week intervention period.

Results: Analysis of data showed that there were significant differences between baseline scores (Headache Frequency Average $=11.47 / \mathrm{mo}$, MIDAS average=25.4) and postintervention follow-up (Headache Frequency Average=6.63/mo, MIDAS average $=11.7$, Frequency $P<.01$, MIDAS $P<.01)$. Postintervention interviews revealed that more than $90 \%$ of the participants felt that the Halo beta app was easy to use and provided a benefit to the user. More than $85 \%$ of participants enjoyed using a daily headache diary and felt it gave them greater insights into their condition. More than $90 \%$ of the patients felt that the relaxation training had helped reduce the frequency of their headaches or headache related disability.

Conclusions: We found that mindfulness and relaxation-based interventions administered through the Halo beta app was a feasible and acceptable treatment for people suffering from frequently recurring headaches and migraines or disability associated with headaches and migraines. The pilot demonstrated the potential feasibility and usability of a mobile health app in delivering behavioral therapy for headache and migraine as an adjunct or alternative to in-office behavioral therapy for headache and migraine.
\end{abstract}

(iproc 2018;4(2):e11814) doi: $10.2196 / 11814$

\section{KEYWORDS}

app; applications; behavioral health; behavioral interventions; chronic conditions; pain; headache; migraine; chronic pain 
Edited by T Hale; this is a non-peer-reviewed article. Submitted 02.08.18; accepted 29.08.18; published 17.09.18.

Please cite as:

Kirell A, Shingleton R

Mobile Behavioral Therapy for Headache: Pilot Study

iproc 2018;4(2):e11814

URL: http://www.iproc.org/2018/2/e11814/

doi: $\underline{10.2196 / 11814}$

PMID:

(C)Adam Kirell, Rebecca Shingleton. Originally published in Iproceedings (http://www.iproc.org), 17.09.2018. This is an open-access article distributed under the terms of the Creative Commons Attribution License (https://creativecommons.org/licenses/by/4.0/), which permits unrestricted use, distribution, and reproduction in any medium, provided the original work, first published in Iproceedings, is properly cited. The complete bibliographic information, a link to the original publication on http://www.iproc.org/, as well as this copyright and license information must be included. 